Artículo Original. Enero-Diciembre 2019; 9:1-11. Recibido: 01/02/2019 Aceptado: 17/06/2019.

http://dx.doi.org/10.21929/abavet2019.914

\title{
Determinación de ácidos grasos no esterificados, $\beta$-hidroxibutirato, triacilglicerol y colesterol durante el balance energético negativo en vacas Holstein
}

\author{
Determination of non-esterified fatty acids, $\beta$-hydroxybutyrate, triacylglycerol and \\ cholesterol during negative energy balance in Holstein cows
}

\author{
Prado-Rebolledo Omar1@ omarpr@ucol.mx, Valpuesta-Santos Gustavo'(C) \\ gvalpuesta0@ucol.mx, Valencia-Magaña Favio² faviovm mvz@hotmail.com, \\ Hernández-Rivera Juan ${ }^{1}$ (i) ihernandez2@ucol.mx, Macedo-Barragán Rafael'10 \\ macedo@ucol.mx, García-Casillas Arturo+1 cesargarciacasillas@hotmail.com
}

${ }^{1}$ Facultad de Medicina Veterinaria y Zootecnia, Universidad de Colima. México. ${ }^{2}$ Estudiante de la Facultad de Medicina Veterinaria y Zootecnia, Universidad de Colima, México. *Autor responsable y de correspondencia: García-Casillas Arturo. Facultad de Medicina Veterinaria y Zootecnia, Universidad de Colima. Kilómetro 40 Carretera Colima-Manzanillo, S/N, Tecomán, Colima, México, CP. 28100. E-mail: cesargarciacasillas@hotmail.com

\section{RESUMEN}

El objetivo del presente trabajo fue cuantificar la concentración de ácidos grasos no esterificados, $\beta$ hidroxibutirato, triacilglicerol y colesterol durante el balance energético negativo, en vacas Holstein. El estudio se llevó a cabo mediante el muestreo y análisis de 250 sueros sanguíneos obtenidos de 50 vacas Holstein. Después del parto, el primer muestreo se realizó a los $7 \mathrm{~d}$, el segundo a los $15 \mathrm{~d}$ y los tres restantes a los 30, 45 y $60 \mathrm{~d}$. Valores medios para ácidos grasos no esterificados, $\beta$-hidroxibutirato, triacilglicerol y colesterol fueron determinados. El conjunto de datos resultante se analizó mediante distribución de Gauss y estadística descriptiva. Se establecieron intervalos de confianza del 95\%. La comparación entre grupos se llevó a cabo por Análisis de Varianza e histograma de frecuencia. Los ácidos grasos no esterificados, el $\beta$-hidroxibutirato y el triacilglicerol, son consistentes con los valores reportados internacionalmente. La concentración de colesterol mostró un valor ligeramente superior a las referencias. Los AGNE no presentaron diferencias entre grupos, el $\beta$-hidroxibutirato registró su valor más alto a los $15 \mathrm{~d}$ posparto, y más bajo a los $60 \mathrm{~d}$ posparto. El triacilglicerol y colesterol mostraron su concentración más alta a los $60 \mathrm{~d}$ posparto, y la más baja a los 15 d posparto. Los intervalos de confianza calculados se podrían utilizar a nivel hato para detectar situaciones de alerta cuando al menos $5 \%$ de las vacas en la muestra se sitúen fuera del intervalo de referencia.

Palabras clave: Perfiles metabólicos, ácidos grasos no esterificados, $\beta$-hidroxibutirato, triacilglicerol, colesterol, vacas lecheras.

\begin{abstract}
The aim of this study was to quantify the concentration of non-esterified fatty acids, $\beta$-hydroxybutyrate, triacylglycerol and cholesterol during the negative energy balance in Holstein cows. The study was carried out by sampling and analyzing 250 blood serums obtained from 50 Holstein cows. After calving, the first sample was performed at $7 d$, the second at $15 d$ and the remaining three at 30,45 and $60 \mathrm{~d}$. Mean values for non-esterified fatty acids, $\beta$-hydroxybutyrate, triacylglycerol, and cholesterol were calculated. The resulting data set was analyzed by Gaussian distribution and descriptive statistics. Confidence intervals of 95\% were established. The comparison between groups was assessed by Analysis of Variance and frequency histogram. The non-esterified fatty acids, $\beta$-hydroxybutyrate, and triacylglycerol are consistent
\end{abstract}


with values reported by international literature. Higher values were found for cholesterol. The AGNE did not present differences between groups. The $\beta$-hydroxybutyrate showed its highest concentration at $15 \mathrm{~d}$ postpartum and the lowest at $60 \mathrm{~d}$ postpartum. Triacylglycerol and cholesterol showed their highest concentration at $60 \mathrm{~d}$ postpartum, and the lowest at $15 \mathrm{~d}$ postpartum. The calculated confidence intervals could be used at herd level to detect alert situations when at least $5 \%$ of the sampled cows would fall outside of the calculated reference interval for a given parameter.

Keywords: Metabolic profiles, non-esterified fatty acids, $\beta$-hydroxybutyrate, triacylglycerol, cholesterol, dairy cow.

\section{INTRODUCCIÓN}

El balance energético negativo (BEN) comprende el déficit entre el consumo de energía por parte del animal y la energía requerida para el mantenimiento, la preñez en la vaca gestante y la energía para la lactación en la vaca lactante (van Hoeij et al., 2017). Durante este periodo, los requerimientos energéticos se incrementan como respuesta al aumento de la producción de leche (PL) (Lucey et al., 2017) y alcanzan su máximo aproximadamente seis semanas posparto (p.p.) (Contreras et al., 2018). Sin embargo, el consumo de materia seca (MS) comienza a disminuir después del parto (Dorea et al., 2017), y el mayor consumo de energía podría no alcanzarse hasta la semana dieciseis p.p. (Sheehy et al., 2017). Por consiguiente, la vaca podría alcanzar un balance energético positivo (BEP) alrededor de los 100 días p.p. (van Hoeij et al., 2017).

EI BEN, conduce a una disminución de glucosa (GLU) e insulina en sangre (Ruoff et al., 2016), y movilización de ácidos grasos no esterificados (AGNE), estos ácidos grasos se utilizan como fuente de energía por la $\beta$-oxidación (Abdelli et al., 2017), pero cuando la movilización de ellos es excesiva, se reestructuran como triacilglicerol (TAG). Unido a este proceso, se activan vías hepáticas alternativas, destacando la formación de cuerpos cetónicos como $\beta$-hidroxibutirato ( $\beta$-HBA) y la formación y almacenamiento hepático de colesterol (COL) (Sun et al., 2016).

Por lo tanto, este proyecto de investigación pretende cuantificar AGNE, $\beta$-HBA, TAG y COL durante el BEN, en vacas Holstein de la granja SEDENA No 1. San Juan, Querétaro, con el fin de identificar su comportamiento a través del tiempo, a partir de mediciones hematoquímicas.

\section{MATERIAL Y MÉTODOS}

Ubicación y animales. El experimento siguió las directrices del Comité Institucional de Cuidado y Uso de Animales de la Universidad de Colima. El estudio se llevó a cabo con 250 sueros sanguíneos, pertenecientes a 50 vacas Holstein, clínicamente sanas, con 660 $\pm 20 \mathrm{~kg}$ de peso vivo $(\mathbf{P V})$ y $26.4 \pm 1.65 \mathrm{~L} / \mathrm{d}$ de leche con $2.88 \pm 0.21 \mathrm{~g} / 100 \mathrm{ml}$ de proteína láctea y $3.37 \pm 0.56 \mathrm{~g} / 100 \mathrm{ml}$ de grasa láctea. Todas las vacas se localizaron en la granja SEDENA No 1.de San Juan, Querétaro, México, a una altitud de 1,856 m. s. n. m., con clima semiárido cálido (Köppen, BSh) (Peel et al., 2007); con una temperatura promedio de $21^{\circ} \mathrm{C}$. Los requerimientos nutricionales de las vacas se estimaron de acuerdo al (NRC, 2001). Las vacas fueron alimentadas dos veces al día con ración totalmente mezclada (RTM) de ensilaje de maíz, heno de alfalfa y semilla de algodón (tabla 1). Recibieron adicionalmente, jabones cálcicos de ácidos grasos destilados de aceite de palma (By Fat; 
NOREL MÉXICO., El Marqués-Querétaro, México) a razón de $400 \mathrm{~g} / \mathrm{d}, 0.3 \mathrm{~kg}$ de bicarbonato de sodio $\left(\mathrm{NaHCO}_{3}\right)$, suplemento mineral con carbonato de calcio, sulfato de magnesio, ortofosfato de calcio, minerales traza (tabla 2) y agua fresca a libre acceso.

Tabla 1. Composición nutricional de la ración totalmente mezclada para vacas en lactancia, granja SEDENA No 1. San Juan, Querétaro

\begin{tabular}{lcc}
\hline Componentes, Materia Seca (MS) & Alimento & Referencia $^{*}$ \\
\hline Proteína Cruda (PC), \% & 16.7 & 14.1 \\
Energía Neta de Lactancia (EN $)$, Mcal/kg & 1.82 & 1.75 \\
Fibra Neutro Detergente (FND), \% & 31.6 & 29.0 \\
Fibra Ácido Detergente (FAD), \% & 22.0 & 19.0 \\
Calcio $\left(\mathrm{Ca}^{2+}\right), \%$ & 1.34 & 0.62 \\
Fósforo $\left(\mathrm{P}^{2+}\right), \%$ & 0.39 & 0.32 \\
Magnesio $\left(\mathrm{Mg}^{2+}\right), \%$ & 0.30 & 0.20 \\
Potasio $\left(\mathrm{K}^{+}\right), \%$ & 1.75 & 1.00 \\
Sodio $\left(\mathrm{Na}^{+}\right), \%$ & 0.45 & 0.22 \\
Zinc $\left(\mathrm{Zn}^{2+}\right), \mathrm{mg} / \mathrm{kg}$ & 58.0 & 43.0 \\
Cobre $\left(\mathrm{Cu}^{2+}\right), \mathrm{mg} / \mathrm{kg}$ & 8.30 & 11.0 \\
Cobalto $\left(\mathrm{Co}^{2+}\right), \mathrm{mg} / \mathrm{kg}$ & 0.70 & 0.11 \\
\hline
\end{tabular}

${ }^{*}$ Requerimientos del NRC para una vaca Holstein con $680 \mathrm{~kg}$ de PV y 25 L/d de producción de leche.

Tabla 2. Composición del suplemento mineral para vacas en lactancia, granja SEDENA No 1. San Juan, Querétaro

\begin{tabular}{lc}
\hline Calcio $\left(\mathrm{Ca}^{2+}\right), \mathrm{g}$ & 96.50 \\
Fósforo $\left(\mathrm{P}^{2+}\right), \mathrm{g}$ & 25.40 \\
Magnesio $\left(\mathrm{Mg}^{2+}\right), \mathrm{g}$ & 14.50 \\
Cloruro de sodio $(\mathrm{NaCl}), \mathrm{g}$ & 48.60 \\
Zinc $\left(\mathrm{Zn}^{2+}\right), \mathrm{mg} / \mathrm{kg}$ & $>25,600$ \\
Cobre $\left(\mathrm{Cu}^{2+}\right), \mathrm{mg} / \mathrm{kg}$ & $>8,800$ \\
Cobalto $\left(\mathrm{Co}^{2+}\right), \mathrm{mg} / \mathrm{kg}$ & $>1,900$ \\
Metionina $(\mathrm{Met}), \mathrm{mg} / \mathrm{kg}$ & $>81,800$ \\
Lisina $(\mathrm{Lis}), \mathrm{mg} / \mathrm{kg}$ & $>37,700$ \\
\hline
\end{tabular}

Muestreo sanguíneo. Al considerar que el pico de PL y el punto máximo de movilización lipídica se localizan de manera común a los 60 d p.p. (Guyot et al., 2017; Contreras et al., 2018). Después del parto, el primer muestreo se realizó a los $7 d$, el segundo a los $15 d$ y los tres restantes a los 30, 45 y $60 \mathrm{~d}$. Las muestras de sangre se obtuvieron mediante punción en la vena coccígea, utilizando tubos al vacío de $8.5 \mathrm{ml}$, con activador de coagulación y gel separador (BD Vacutainer 367988; Becton- Dickinson Co., Franklin Lakes, United States), después del primer ordeño de la mañana y antes de la alimentación. Para la obtención del suero, las muestras se centrifugaron directamente en los establos, a 1,500 x g durante 10 min, mediante una centrífuga portátil (Porta-Spin C828; ÚNICO., Dayton, United States). Posteriormente, los sueros se separaron utilizando tubos de $5 \mathrm{ml}$ con tapa (Tubes 933008; Eppendorf., Madrid, España), fueron congelados a $-20{ }^{\circ} \mathrm{C}$ (Biomedical Freezer MDF-U5412H-PE; PHC Europe B.V., Ámsterdam, Holanda) y transportados con gel refrigerante durante un periodo máximo de 
2 h, en una hielera flexible de 18 L (Cooler Flex Go M5644-710; The Coleman Company, Kansas, United States), donde el suero permaneció congelado hasta su análisis.

Determinación de analitos bioquímicos. La concentración de AGNE, $\beta$-HBA, TAG y COL se determinó por espectrofotometría (Biochemistry Analyzer ES-218 UV-Vis; KONTROLab., Guidonia, Italia). Los analitos bioquímicos, el método analítico de cada parámetro, las unidades en que se expresaron los resultados y los reactivos comerciales correspondientes, se describen en la tabla 3.

Tabla 3. Analitos bioquímicos, unidades, métodos y reactivos comerciales correspondientes

\begin{tabular}{llll}
\hline Analito & Unidad & Método & Reactivo \\
\hline Ácidos grasos no esterificados (AGNE) & $\mathrm{mM}$ & Enzimático $^{\mathrm{a}}$ & $\mathrm{FA} 115^{2}$ \\
$\beta$-hidroxibutirato ( $\beta$-HBA) & $\mathrm{mM}$ & Enzimático $^{\mathrm{b}}$ & $\mathrm{RB} 1007^{2}$ \\
Triacilglicerol (TAG) & $\mathrm{mM}$ & Colorimétrico. Líquido $^{c}$ & $41032^{1}$ \\
Colesterol (COL) & $\mathrm{mM}$ & Colorimétrico $^{{ }^{\circ}}$ & $41020^{1}$
\end{tabular}

aSintetasa-Oxidasa-Peroxidasa; ${ }^{\mathrm{b}} \beta$-hidroxibutirato deshidrogenasa; ${ }^{\mathrm{C}} \mathrm{Glicerolfosfato}$ deshidrogenasa-peroxidasa; ${ }^{\mathrm{d}} \mathrm{Colesterol}$ oxidasa-peroxidasa; ${ }^{1}$ Spinreact., Gerona, España; ${ }^{2}$ Randox Laboratories., Irlanda del Norte, Reino Unido.

El espectro electromagnético se escaneó de acuerdo a la ley de Beer-Lambert, cuya ecuación es:

$$
\frac{I_{t}}{I_{0}}=e^{-k l c}
$$

donde:

$I_{t} \quad=\quad$ rango de luz captado por el tubo de fotocolorimetría;

$I_{0} \quad=\quad$ rango de luz que sale del tubo de fotocolorimetría;

$k=$ coeficiente de extinción molar;

$l=$ longitud $(\mathrm{cm})$ del tubo de fotocolorimetría; $\mathrm{y}$

$c=$ concentración de la muestra ubicada en el tubo de fotocolorimetría.

La precisión y la veracidad para la reproducibilidad de las técnicas, se controló mediante la utilización de suero de control liofilizado (SPINTROL NORMAL 1002100; Spinreact., Girona, España) y (Assayed Bovine Multi-Sera AL 1027; Laboratorios Randox, Irlanda del Norte, Reino Unido). La hemólisis del suero se registró en una escala cualitativa de 0 (nada) a 3 (oscuro). Sueros con interferencia por hemólisis con puntuación 2 o superior, fueron rechazados.

Análisis estadístico. Para el tratamiento de los datos obtenidos se utilizó el programa estadístico (SAS, System, v. 9.0.2, Cary, NC). Los intervalos de confianza del 95\%, siguieron la recomendación de la Federación Internacional de Química Clínica (Solberg, 1987). Se determinó la presencia de datos atípicos para cada analito. Todos los valores con \pm 3 desviaciones estándar (DE) de la media fueron descartados. El conjunto de datos 
resultante se analizó mediante distribución de Gauss, y los percentiles: $\mathrm{P}_{10}-\mathrm{P}_{90}$ y $\mathrm{P}_{25}-\mathrm{P}_{75}$ fueron determinados por (PROC UNIVARIATE; SAS, 2010). La comparación entre grupos (7, 15, 30, 45 y 60 d p.p.), se llevó a cabo por (PROC ANOVA; SAS, 2010) e histograma de frecuencia. Cuando se encontró efecto significativo por grupo $(P<0.05)$, se realizó la prueba de comparación múltiple de Tukey.

\section{RESULTADOS}

Los estadísticos descriptivos para: AGNE, $\beta$-HBA, TAG y COL, determinado a partir de 250 sueros sanguíneos de vacas Holstein, y sus respectivos valores de referencia internacionales se muestran en la tabla 4.

Tabla 4. Media ( $x$ ), desviación estándar (DE), valor de referencia, intervalo de confianza (IC) y percentiles $\left(P_{10}-P_{90}\right)$ y $\left(P_{25}-P_{75}\right)$ para diferentes analitos bioquímicos, $n=250$ sueros sanguíneos de vacas Holstein ${ }^{a}$

\begin{tabular}{lccccc}
\hline Analito & $\boldsymbol{x} \pm \mathbf{D E}$ & Referencia $^{\mathrm{b}}$ & $\mathbf{I C}^{\mathrm{c}}$ & $\mathbf{P}_{\mathbf{1 0}^{-}}-\mathbf{P}_{\mathbf{9 0}}$ & $\mathbf{P}_{\mathbf{2 5}}-\mathbf{P}_{\mathbf{7 5}}$ \\
\hline Ácidos grasos no esterificados (AGNE) & $0.10 \pm 0.04$ & $0.40 \pm 0.20$ & $0.10-0.11$ & $0.05-0.16$ & $0.10-0.11$ \\
$\beta$-hidroxibutirato (mM) & $0.49 \pm 0.16$ & $0.41 \pm 0.03$ & $0.47-0.51$ & $0.28-0.73$ & $0.39-0.59$ \\
Triacilglicerol (mM) & $0.11 \pm 0.03$ & $0.10 \pm 0.10$ & $0.10-0.11$ & $0.06-0.16$ & $0.09-0.14$ \\
Colesterol (mM) & $3.94 \pm 1.15$ & $2.59 \pm 0.51$ & $3.79-4.08$ & $2.50-5.57$ & $3.08-4.72$
\end{tabular}

${ }^{a} 660 \pm 20 \mathrm{~kg}$ de peso vivo (PV) y $26.4 \pm 1.65 \mathrm{~L} / \mathrm{d}$ de leche, con $2.88 \pm 0.21 \mathrm{~g} / 100 \mathrm{~mL}$ de proteína láctea y $3.37 \pm 0.56 \mathrm{~g} / 100 \mathrm{~mL}$ de grasa láctea; ${ }^{b}($ Kaneko et al., 2008); cintervalo de confianza al $95 \%$.

La concentración de AGNE, $\beta$-HBA y TAG, es consistente con los valores reportados internacionalmente. El COL mostró un valor ligeramente superior a la referencia. Los AGNE no presentaron diferencias entre grupos, el $\beta$-HBA registró su valor más alto $(0.74$ $\pm 0.08 \mathrm{mM}$ ) a los $15 \mathrm{~d}$ p.p., (tabla 5); su concentración más baja $(0.27 \pm 0.06 \mathrm{mM})$, se cuantificó a los 60 d p.p. El TAG mostró su concentración más alta $(0.16 \pm 0.01 \mathrm{mM})$ a los 60 d p.p; su valor más bajo $(0.05 \pm 0.01 \mathrm{mM})$, se registró a los 15 d p.p. La concentración de COL, cuantificó su valor más alto $(5.58 \pm 0.39 \mathrm{mM})$ a los 60 d p.p; su concentración más baja $(2.32 \pm 0.51 \mathrm{mM})$, se registró a los 15 d p.p.

Tabla 5. Comparación de diferentes analitos bioquímicos, durante diferentes días posparto,

\begin{tabular}{lccccc}
\multicolumn{1}{c}{$\boldsymbol{n}=\mathbf{5 0}$ vacas Holstein/grupo } \\
\hline & $\mathbf{7}$ & $\mathbf{1 5}$ & $\mathbf{3 0}$ & $\mathbf{4 5}$ & $\mathbf{6 0}$ \\
\cline { 2 - 6 } Analito & \multicolumn{5}{c}{ Días posparto } \\
\hline Ácidos grasos no esterificados (mM) & $0.13 \pm 0.05^{\mathrm{a}}$ & $0.14 \pm 0.06^{\mathrm{a}}$ & $0.12 \pm 0.02^{\mathrm{a}}$ & $0.12 \pm 0.03^{\mathrm{a}}$ & $0.12 \pm 0.01^{\mathrm{a}}$ \\
$\beta$-hidroxibutirato (mM) & $0.57 \pm 0.02^{\mathrm{a}}$ & $0.74 \pm 0.08^{\mathrm{b}}$ & $0.48 \pm 0.02^{\mathrm{c}}$ & $0.40 \pm 0.01^{\mathrm{d}}$ & $0.27 \pm 0.06^{\mathrm{e}}$ \\
Triacilglicerol (mM) & $0.11 \pm 0.01^{\mathrm{a}}$ & $0.05 \pm 0.01^{\mathrm{b}}$ & $0.09 \pm 0.01^{\mathrm{c}}$ & $0.12 \pm 0.01^{\mathrm{a}, \mathrm{d}}$ & $0.16 \pm 0.01^{\mathrm{e}}$ \\
Colesterol (mM) & $3.94 \pm 0.18^{\mathrm{a}}$ & $2.32 \pm 0.51^{\mathrm{b}}$ & $3.28 \pm 0.22^{\mathrm{c}}$ & $4.56 \pm 0.20^{\mathrm{d}}$ & $5.58 \pm 0.39^{\mathrm{e}}$
\end{tabular}

"Dentro de renglones valores con diferente letra difieren significativamente entre si $(\mathrm{P}<0.05)$. Todos los datos son presentados por media $\pm D E$. $(P<0.05)$. 


\section{DISCUSIÓN}

Durante el BEP, las vacas lecheras de alto valor genético, tienen una baja movilización lipídica y su concentración de AGNE es aproximadamente $0.25 \mathrm{mM}$ (da Fonseca et al., 2004). Concentraciones superiores a $0.40 \mathrm{mM}$ indican BEN y una elevada movilización lipídica (Oetzel, 2004). De acuerdo con esta referencia, ninguna de las vacas muestreadas presentó evidencia de una elevada movilización de lípidos, porque sólo tres vacas superaron los $0.25 \mathrm{mM}$ para AGNE. En los últimos años para discriminar entre vacas sanas y vacas afectadas por cetosis subclínica, se han utilizado diferentes puntos de corte entre 1 y $1.4 \mathrm{mM}$, con respecto a las concentraciones séricas de $\beta$-HBA (Contreras et al., 2018).

En el presente estudio, los AGNE no presentaron diferencias entre grupos, pero se observó un incremento de $\beta$-HBA a los 15 d p.p. (figura 1), y después se cuantificó un descenso progresivo conforme avanzó la lactancia.

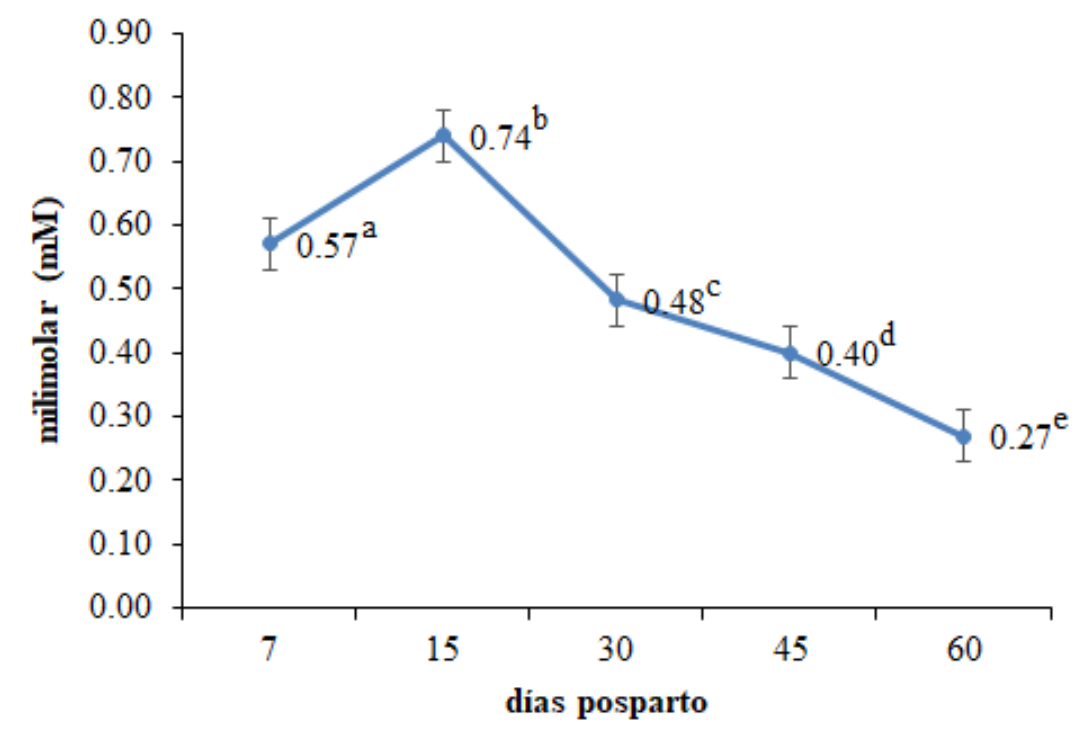

Se obtuvieron diferencias significativas entre los grupos indicados con letras

diferentes $(P<0.05)$. Todos los datos son presentados por media $\pm D E$.

Figura 1. Comparación de $\beta$-hidroxibutirato a través del tiempo, $n=50$ vacas Holstein

Estos datos sugieren que la concentración de AGNE podría ser un indicador de cetosis subclínica, menos eficiente en comparación con el $\beta$-HBA. Suposición consistente con lo reportado por Ruoff et al. (2016) y Abdelli et al. (2017), quienes indicaron que durante el periodo seco, el uso de AGNE es un mejor indicador de BEN en comparación con el $\beta$ HBA, pero que en vacas p.p., el $\beta$-HBA es más útil.

El BEN presenta una inadecuada biosíntesis de GLU, acompañada de una menor estimulación de las células $\beta$ del páncreas, comprometiendo la liberación de insulina (Abdelli et al., 2017). En consecuencia, durante las primeras semanas p.p., se presenta una marcada hipoglucemia (Jump, 2011). El metabolismo de las vacas lecheras, responde a esta situación con la hidrólisis de TAG almacenados en el tejido adiposo, 
obteniendo AGNE que pueden ser oxidados dentro de la matriz mitocondrial hepática (García et al., 2015), para la producción de acetil-Coenzima A (Wakil y Abu-Elheiga, 2009). Durante la condensación consecutiva de acetil-Coenzima $A$, se obtiene acetoacetato (AcAc), como producto alterno (Ruoff et al., 2016), y una parte de él se reduce por la $\beta$-hidroxibutirato deshidrogenasa a $\beta$-HBA (García et al., 2015). Por lo tanto, durante los primeros días de lactancia su concentración se eleva ligeramente en respuesta a la movilización lipídica, y desciende cuando el balance energético se vuelve positivo (Sun et al., 2016). Esta información es congruente, con los resultados aquí obtenidos (figura 1), ya que se observó el incremento de este analito hasta los 15 d p.p., y después se cuantificó un descenso progresivo conforme avanzó la lactancia.

Durante el BEN propio de la lactancia temprana, las reservas de grasa corporal se movilizan y los AGNE transportados por albumina (ALB) (Imhasly et al., 2015), llegan al hígado donde se oxidan o se esterifican en TAG (Jump, 2011). La acumulación temporal de TAG en el hígado de vacas lecheras es un proceso fisiológico normal (Argüeso et al., 2011). Todas las vacas tienen un grado moderado de lipidosis hepática después del período seco, y acumulan TAG en el hígado durante los primeros días p.p. (Abdelli et al., 2017). Por lo tanto, esta acumulación es parcialmente responsable de la baja concentración de TAG a los 15 y 30 d en el torrente sanguíneo (figura 2).

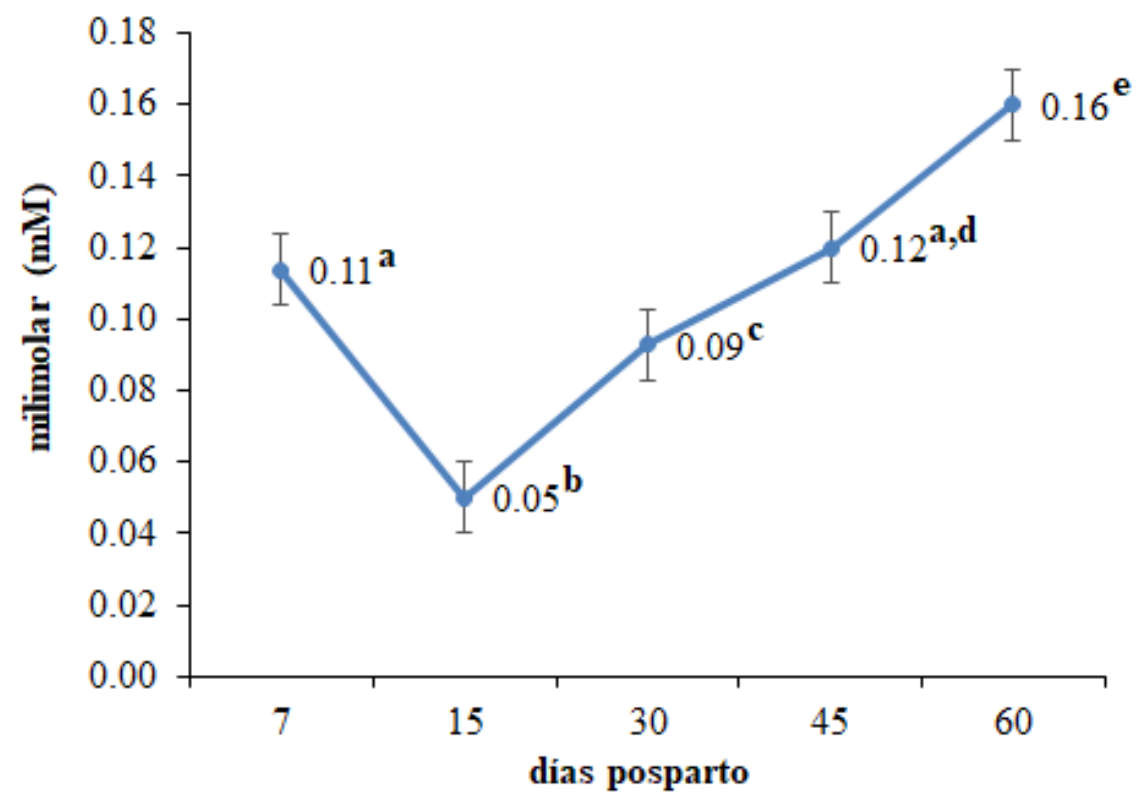

Se obtuvieron diferencias significativas entre los grupos indicados con letras diferentes $(P<0.05)$. Todos los datos son presentados por media $\pm D E$.

Figura 2. Comparación de triacilgliceroles a través del tiempo, $n=50$ vacas Holstein

Con respecto al COL y su concentración ligeramente por encima de la referencia, vale la pena considerar los aportes nutricionales, ya que los alimentos altos en grasa pueden elevar la concentración de COL. Es más, Duske et al. (2009) y Zárate-Martínez et al. 
(2011) indicaron que las vacas lecheras suplementadas con grasas inertes en rumen, presentaron un ligero incremento en la concentración sérica de COL. Esta información es consistente con nuestros resultados, ya que a las vacas del estudio se les proporcionaba jabones cálcicos de ácidos grasos destilados de aceite de palma (By Fat; NOREL MÉXICO., El Marqués Querétaro, México) a razón de 400 g/d.

Por otro lado, es oportuno señalar que sólo una pequeña parte del COL contenido en la leche se origina de novo en la glándula mamaria; mientras que la captación de COL sanguíneo durante las primeras semanas p.p., es mucho más acentuada (Folnožić et al., 2015). Al respecto, Mani et al. (2009) y Mani et al. (2011) señalaron que los transcritos de ácido ribonucleico mensajero (ARNm) de los transportadores ABCA1 y ABCG1, encargados de regular el ingreso de fosfolípidos y $\mathrm{COL}$ al interior de los lactocitos, exhiben patrones diferentes a través de la lactancia, cuantificando su mayor expresión antes de los 20 d p.p. Por tal motivo, el descenso de COL en el torrente sanguíneo a los 15 d p.p. (figura 3) podría ser explicado por un aumento del flujo de COL a la glándula mamaria, producto de la mayor expresión de los transportadores ABCA1 y ABCG1.

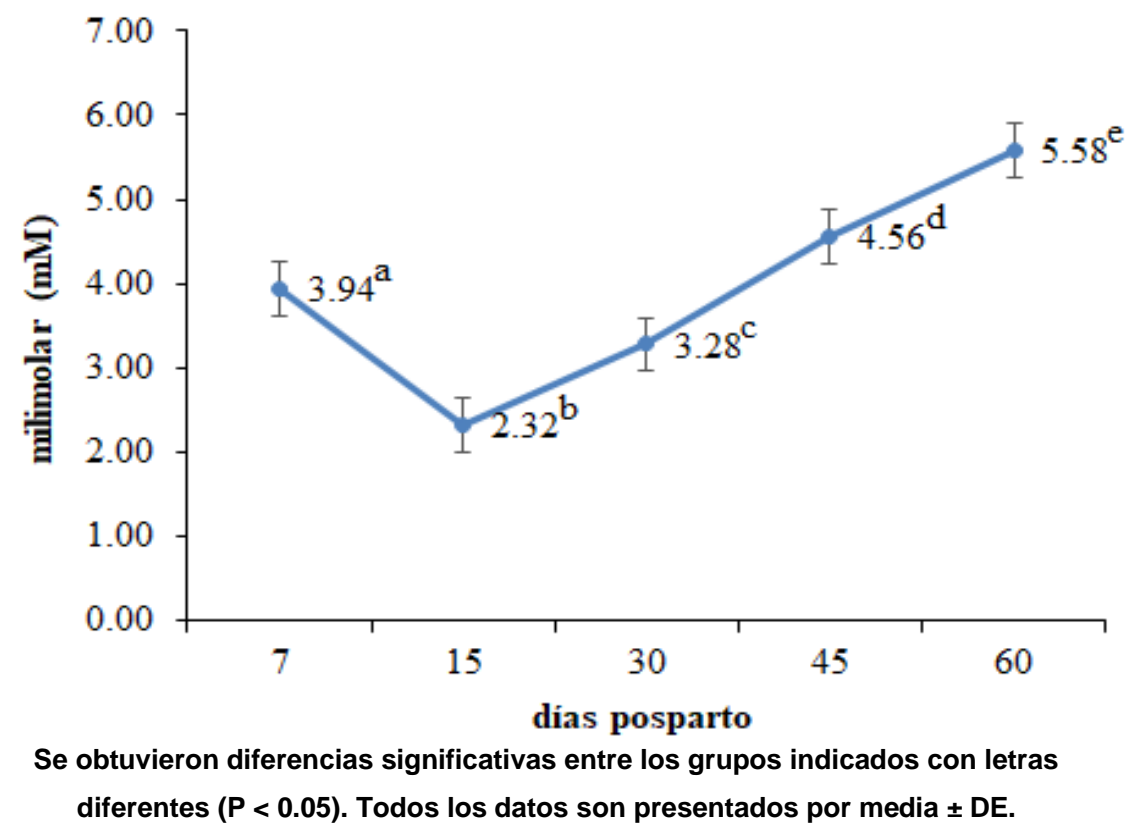

Figura 3. Comparación de colesterol a través del tiempo, $n=50$ vacas Holstein

\section{CONCLUSIONES}

La concentración de AGNE, $\beta$-HBA y TAG, es consistente con los valores reportados internacionalmente. La concentración de COL $(3.94 \pm 0.18 \mathrm{mM})$, mostró un valor ligeramente superior a las referencias. Los AGNE no presentaron diferencias entre grupos (7, 15, 30, 45 y 60 d p.p.); el $\beta$-HBA registró su valor más alto $(0.74 \pm 0.08 \mathrm{mM})$ a los 15 d p.p., y más bajo $(0.27 \pm 0.06 \mathrm{mM})$ a los $60 \mathrm{~d}$ p.p. Los TAG y el COL mostraron 
su concentración más alta $(0.16 \pm 0.01 \mathrm{mM}$ y $5.58 \pm 0.39 \mathrm{mM}$ respectivamente $)$ a los 60 d p.p., y la más baja $(0.05 \pm 0.01 \mathrm{mM}$ y $2.32 \pm 0.51 \mathrm{mM}$ respectivamente) a los $15 \mathrm{~d}$ p.p. Los intervalos de confianza calculados se podrían utilizar a nivel hato para detectar situaciones de alerta, cuando al menos $5 \%$ de las vacas; en la muestra se sitúen fuera del intervalo de referencia.

\section{AGRADECIMIENTOS}

Este proyecto fue apoyado por el Consejo Nacional de Ciencia y Tecnología de México (CONACyTMéxico).

\section{LITERATURA CITADA}

ABDELLI A, Raboisson D, Kaidi R, Ibrahim B, Kalem A, Iguer-Ouada M. 2017. Elevated non-esterified fatty acid and $\beta$-hydroxybutyrate in transition dairy cows and their association with reproductive performance and disorders: a meta-analysis. Theriogenology. 93(1):99-104. ISSN: 1879-3231 (Electronic) 0093-691X (Linking). DOI: 10.1016/j.theriogenology.2017.01.030.

ARGÜESO AR, Díaz DJL, Díaz PJA, Rodríguez GA, Castro MM, Diz LF. 2011. Lipids, cholesterol and lipoproteins. Galicia Clin. 72(suppl):7-17. ISSN: 0304-4866. DOI: Not available.

CONTRERAS GA, Strieder BC, De Koster J. 2018. Modulating adipose tissue lipolysis and remodeling to improve immune function during the transition period and early lactation of dairy cows. J Dairy Sci. 101(3):2737-2752. ISSN: 1525-3198 (Electronic) 0022-0302 (Linking). DOI: 10.3168/jds.2017-13340.

DA FONSECA LLF, Rodríguez MPH, dos Santos VM, Lima PA, Lucci SC. 2004. Supplementation of dairy cows with propylene glycol during the periparturient period: effects on body condition score, milk yield, first estrus post-partum, $\beta$-hydroxybutyrate, non-esterified fatty acids and glucose concentrations. Cienc Rural. 34(3):897-903. ISSN: 1678-4596 (Electronic). DOI: 10.1590/S0103-84782004000300037.

DOREA JRR, French EA, Armentano LE. 2017. Use of milk fatty acids to estimate plasma nonesterified fatty acid concentrations as an indicator of animal energy balance. J Dairy Sci. 100(8):6164-6176. ISSN: 1525-3198 (Electronic) 0022-0302 (Linking). DOI: 10.3168/jds.2016-12466.

DUSKE K, Hammon HM, Langhof AK, Bellmann O, Losand B, Nurnberg K, Nurnberg G, Sauerwein H, Seyfert HM, Metges CC. 2009. Metabolism and lactation performance in dairy cows fed a diet containing rumen-protected fat during the last twelve weeks of gestation. J Dairy Sci. 92(4):1670-1684. ISSN: 1525-3198 (Electronic) 0022-0302 (Linking). DOI: 10.3168/jds.2008-1543.

FOLNOŽIĆ I, Turk R, Đuričić D, Vince S, Pleadin J, Flegar-Meštrić Z, Valpotić H, Dobranić T, Gračner D, Samardžija M. 2015. Influence of Body Condition on Serum Metabolic Indicators of Lipid Mobilization and Oxidative Stress in Dairy Cows During the Transition 
Period. Reprod Domest Anim. 50(6):910-917. ISSN: 1439-0531 (Electronic) 0936-6768 (Linking). DOI: 10.1111/rda.12608.

GARCÍA CAC, Montiel RLA, Borderas TF, Girard V. 2015. Relationship between $\beta$ hydroxybutyrate and fat:protein ratio of milk during early lactation in dairy cows. Arch Med Vet 47(1):21-25. ISSN: 0719-8132 (Electronic) 0719-8000 (Linking). DOI: 10.4067/S0301732X2015000100005.

GUYOT H, Detilleux J, Lebreton P, Garnier C, Bonvoisin M, Rollin F, Sandersen C. 2017. Comparison of various indices of energy metabolism in recumbent and healthy dairy cows. PLoS One. 12(1):e0169716-e0169726. ISSN: 1932-6203 (Electronic) 1932-6203 (Linking). DOI: 10.1371/journal.pone.0169716.

IMHASLY S, Bieli C, Naegeli H, Nystrom L, Ruetten M, Gerspach C. 2015. Blood plasma lipidome profile of dairy cows during the transition period. BMC Vet Res. 11(1):252-259. ISSN: 1746-6148 (Electronic) 1746-6148 (Linking). DOI: 10.1186/s12917-015-0565-8.

JUMP DB. 2011. Fatty acid regulation of hepatic lipid metabolism. Curr Opin Clin Nutr Metab Care. 14(2):115-120. ISSN: 1473-6519 (Electronic) 1363-1950 (Linking). DOI: 10.1097/MCO.0b013e328342991c.

KANEKO JJ, Harvey WJ, Bruss LM. 2008. "Appendix VIII Blood Analyte Reference Values in Large Animals". In: Kaneko JJ, Harvey WJ , Bruss LM, Clinical Biochemistry of Domestic Animals. California, Estados Unidos: Academic Press. 882-888 p. ISBN: 9780-12-370491-7.

LUCEY JA, Otter D, Horne DS. 2017. A 100 year review: progress on the chemistry of milk and its components. J Dairy Sci. 100(12):9916-9932. ISSN: 1525-3198 (Electronic) 0022-0302 (Linking). DOI: 10.3168/jds.2017-13250.

MANI O, Sorensen MT, Sejrsen K, Bruckmaier RM, Albrecht C. 2009. Differential expression and localization of lipid transporters in the bovine mammary gland during the pregnancy-lactation cycle. J Dairy Sci. 92(8):3744-3756. ISSN: 1525-3198 (Electronic) 0022-0302 (Linking). DOI: 10.3168/jds.2009-2063.

MANI O, Korner M, Ontsouka CE, Sorensen MT, Sejrsen K, Bruckmaier RM, Albrecht C. 2011. Identification of ABCA1 and ABCG1 in milk fat globules and mammary cells-implications for milk cholesterol secretion. J Dairy Sci. 94(3):1265-1276. ISSN: 1525-3198 (Electronic) 0022-0302 (Linking). DOI: 10.3168/jds.2010-3521.

NRC. 2001. National Research Council. Nutrient Requirements of Dairy Cattle. 7th ed. National Academy Press, Washington DC., USA.

OETZEL GR. 2004. Monitoring and testing dairy herds for metabolic disease. Vet Clin North Am Food Anim Pract 20(3):651-674. ISSN: 0749-0720 (Electronic). DOI: 10.1016/j.cvfa.2004.06.006. 
PEEL MC, Finlayson BL, McMahon TA. 2007. Updated world map of the Köppen-Geiger climate classification. Hydrol Earth Syst Sci. 11(5):1633-1644. ISSN: 1027-5606 (Electronic) 1607-7938 (Linking). DOI: 10.5194/hess-11-1633-2007, 2007.

RUOFF J, Bertulat S, Burfeind O, Heuwieser W. 2016. Associations of $\beta$-hydroxybutyrate, cholesterol, triglycerides and high-density lipoproteins to non-esterified fatty acids preand postpartum. J Dairy Res. 83(4):447-452. ISSN: 1469-7629 (Electronic) 0022-0299 (Linking). DOI: 10.1017/S0022029916000534.

SAS Institute. 2010. Statistical Analysis Software SAS/STAT®. version 9.0.2, Cary, N.C., USA: SAS Institute Inc., ISBN: 978-1-60764-599-3. Available: http://www.sas.com/en us/software/analytics/stat.html

SHEEHY MR, Fahey AG, Aungier SPM, Carter F, Crowe MA, Mulligan FJ. 2017. A comparison of serum metabolic and production profiles of dairy cows that maintained or lost body condition 15 days before calving. J Dairy Sci. 100(1):536-547. ISSN: 1525-3198 (Electronic) 0022-0302 (Linking). DOI: 10.3168/jds.2016-11206.

SOLBERG HE. 1987. Approved recommendation (1987) on the theory of reference values. Part 5. Statistical treatment of collected reference values. Determination of reference limits. Clin Chim Acta. 170(2-3):S13-S32. ISSN:1873-3492 (Electronic) 00098981 (Linking) DOI: 10.1016/0009-8981(87)90151-3.

SUN F, Cao Y, Cai C, Li S, Yu C, Yao J. 2016. Regulation of nutritional metabolism in transition dairy cows: energy homeostasis and health in response to post-ruminal choline and methionine. PLoS One. 11(8):e0160659-e0160669. ISSN: 1932-6203 (Electronic) 1932-6203 (Linking). DOI: 10.1371/journal.pone.0160659.

VAN HOEIJ RJ, Dijkstra J, Bruckmaier RM, Gross JJ, Lam T, Remmelink GJ, Kemp B, van Knegsel ATM. 2017. The effect of dry period length and postpartum level of concentrate on milk production, energy balance, and plasma metabolites of dairy cows across the dry period and in early lactation. J Dairy Sci. 100(7):5863-5879. ISSN: 15253198 (Electronic) 0022-0302 (Linking). DOI: 10.3168/jds.2016-11703.

WAKIL SJ, Abu-Elheiga LA. 2009. Fatty acid metabolism: target for metabolic syndrome. $J$ Lipid Res 50(Suppl):138-143. ISSN: $1539-7262$ (Electronic) 0022-2275 (Linking). DOI: 10.1194/jlr.R800079-JLR200.

ZÁRATE-MARTÍNEZ JP, Vinay-Vadillo JC, Carballo CO, Hernández-Hernández VD, Amezcua-Manjarres EV. 2011. Efecto de la alimentación con grasas protegidas en vacas de doble propósito. Agron Mesoam. 22(2):359-366. ISSN: 1021-7444. 